\title{
Chemical Segregation and Microstructural Evolution of Fiber Laser-Welded Low Carbon Sheet Steel
}

\author{
Ann N. Chiaramonti ${ }^{1}$, Stephanie L. Miller ${ }^{1}$, Paul T. Blanchard ${ }^{2}$, and Erik Pfeif ${ }^{1}$ \\ 1. Material Measurement Laboratory, National Institute of Standards and Technology, Boulder USA. \\ 2. Physical Measurement Laboratory, National Institute of Standards and Technology, Boulder USA.
}

Laser beam welding (LBW) has significantly improved since its first commercial deployment in 1966, and is the preferred joining method when high weld quality, high production speed, and low thermal distortion are required. LBW encompasses a wide range of similar and dissimilar materials as is often the case in the automotive, aerospace, and medical industries. However this technology has not been implemented in many structurally critical applications, i.e., automotive chassis components, despite 50 years of development work and documentation in the literature. NIST has undertaken a multi-part initiative to support extending LBW to structurally critical components, with the current work focusing on nano-scale characterization by electron microscopy, diffraction, and atom probe tomography.

In the automotive sector, much of the fundamental LBW literature relies on evaluation of macroscopic weld quality coupled with mechanical properties assessment. However, more rigorous and fundamental examination of the phase transformations is needed to fully optimize laser processing for complex microstructures such as those associated with HSLA, dual-phase, TRIP, and TWIP steels such as is often performed during heat treatment studies [1]. Subtle differences in sub-micron scale local chemistry and/or microstructure morphology can dramatically affect mechanical behavior, driving the need to reliably characterize these differences by scanning electron microscopy (SEM), transmission electron microscopy (TEM) and electron backscatter diffraction (EBSD). Furthermore, atom probe tomography (APT) provides a tool for direct measurement of local chemistry and boundary segregation. Atomic mobilities and kinetic diffusion rates and can be inferred with APT measurements, providing critical parameters for predictive modeling. Accurate predictive modeling would allow for tailoring weld parameters to achieve optimal phase distributions, thus reproducibly controlling mechanical performance of the welds.

The difference in hardness between the base material and the weld metal is often used as a criterion for weld quality assessment. The weld centerline represents the last liquid to solidify. This region develops the highest internal temperature gradients, and likely shows the greatest deviation from thermodynamic equilibrium. Thus the lath martensite structure identified at the center of the weld metal was investigated in more detail. From a phase transformation perspective, whether the interlath areas are made up of carbide or retained austenite is indicative of the kinetics of the rapid solidification process that occurs during LBW; This in turn affects the mechanical properties of the martensite. Also, confirmation of carbon clustering or full auto-tempering of the martensite provides insight into the non-equilibrium solidification conditions affecting the final mechanical properties.

The goal of this study was to provide fundamental data for phase transformation prediction during the rapid solidification of LBW material, and information on how processing controls the microstructure and mechanical properties for a commercially relevant medium-carbon sheet steel. The results presented here target the nature of the interlath regions and intralath precipitation. Findings can strengthen 
modeling efforts by incorporating new kinetic and thermodynamic data based on real-world observations.

[1] D. Raabe et al., Acta Materialia 61 (2013) 6132.

[2] This work is a contribution of the U.S. Department of Commerce and is not subject to copyright in the United States.
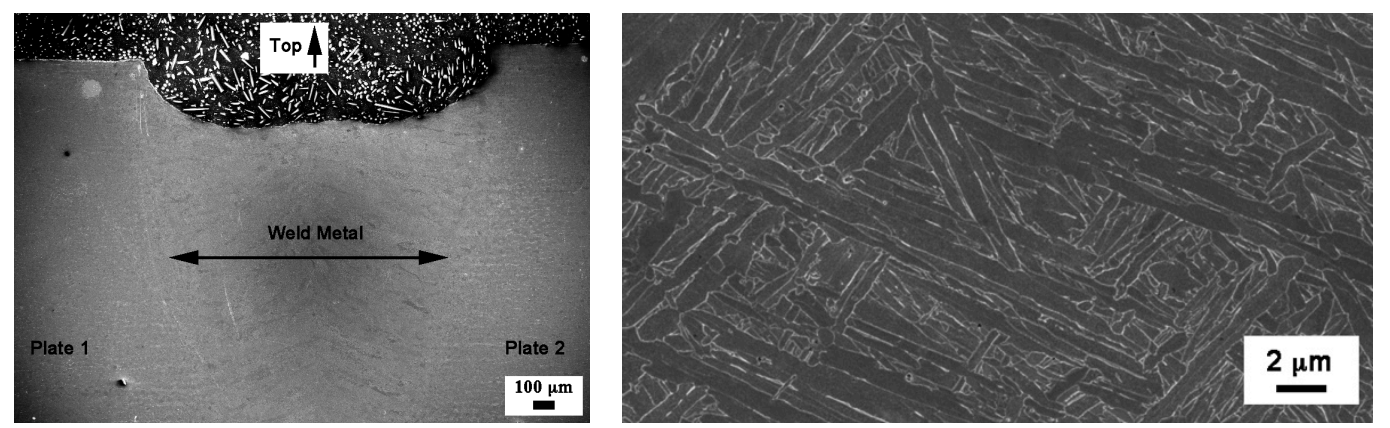

Figure 1. (L) SEM image of the laser butt weld of interest. (R) Higher magnification SEM image of the weld metal showing martensite lath structure with an interlath phase revealed after light Nital etching.

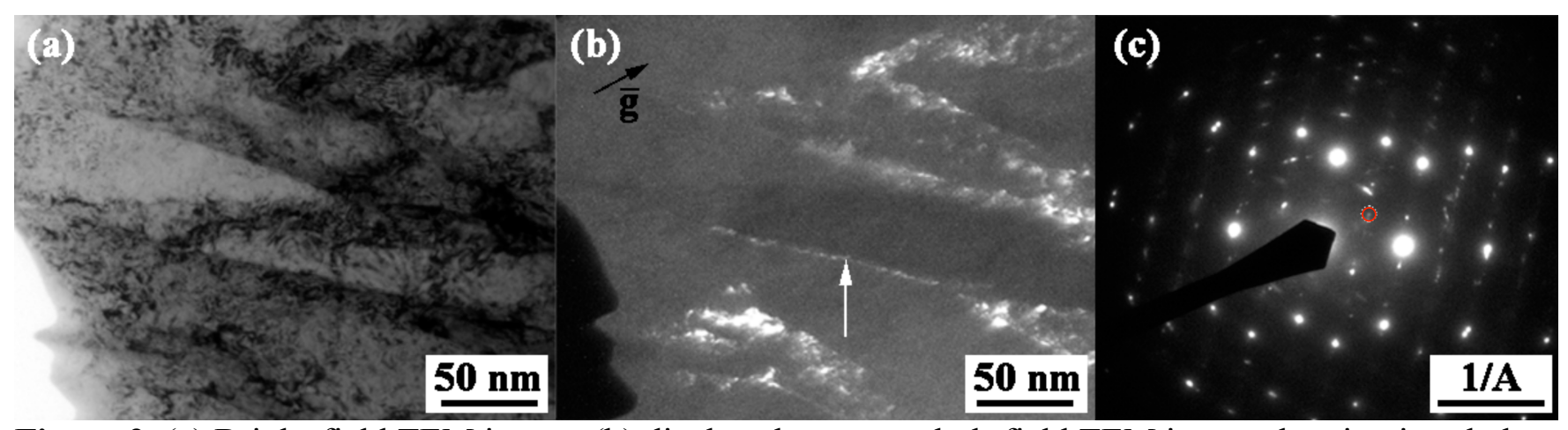

Figure 2. (a) Bright-field TEM image, (b) displaced-aperture dark-field TEM image showing interlath phase (white arrow). (c) Selected area diffraction pattern corresponding to the images in a \& $b$. The diffraction spot used to make the dark-field image is indicated.

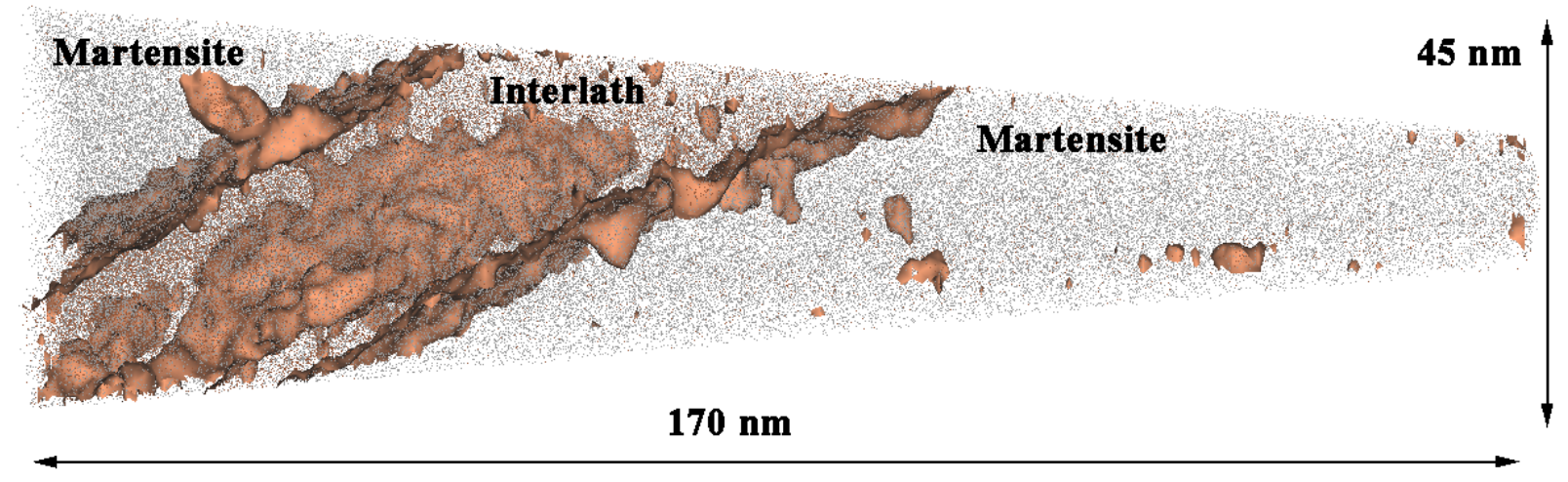

Figure 3. APT reconstruction of the weld metal showing martensite and interlath material. Iron atoms are shown in gray, carbon in orange. A 2.6 atomic percent $\mathrm{C}$ isoconcentration surface is shown. 